\title{
The Effects of Cognitive Training on Healthy Elderly Thais: Randomized Controlled Trial
}

\author{
Muthita Phanasathit ${ }^{1,2}$, Chaichana Nimnuan ${ }^{3}$, Vitool Lohsoonthorn ${ }^{2}$ \\ ${ }^{1}$ Department of Psychiatry, Center of Excellence of Applied Epidemiology, Faculty of Medicine, Thammasat University, Thailand \\ 2 Department of Preventive and Social Medicine, Faculty of Medicine, Chulalongkorn University, Thailand \\ ${ }^{3}$ Department of Psychiatry, Faculty of Medicine, Chulalongkorn University, Thailand
}

\section{Objective:} low-technology devices in healthy elderly Thais in community-based populations.

\section{Methods:}

This study was a two-arm parallel single-blinded randomized controlled intervention trial. Eighty-six qualified participants aged at least 55 years old were recruited from an elderly club in Bangkok suburbs.

The exercises in the CTI combined with complex attention, executive function, learning and memory, language, visuospatial ability, and social cognition. The CTI was run by an audio-visual digital video disk which explained instruction and demonstration in each lesson and involved an individual paper-pencil workbook. The CTI was implemented twice per week with 30 minutes allocated to each session during 12 weeks.

\section{Enrollment \\ Assessed for eligibility $(n=88)$}

Met exclusion criteria $(n=2)$

- Schizophrenia $(n=1)$

- Cerebrovascular disease $(n=1)$

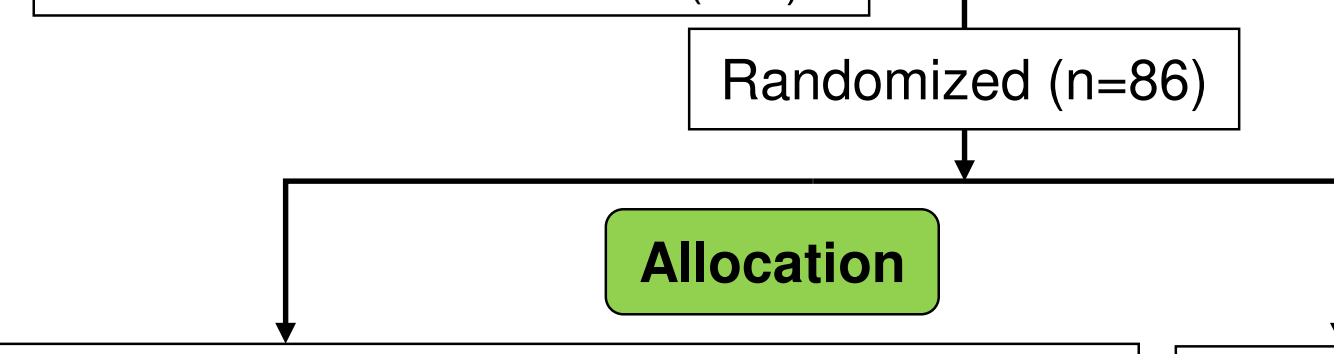

Cognitive training intervention $(n=44)$

- Received allocated intervention $(n=37)$

- Did not receive allocated intervention $(n=7)$

- Declined to participate $(n=5)$

- Having hospitalization and surgery $(n=1)$

- Unable to be contacted by phone $(n=1)$

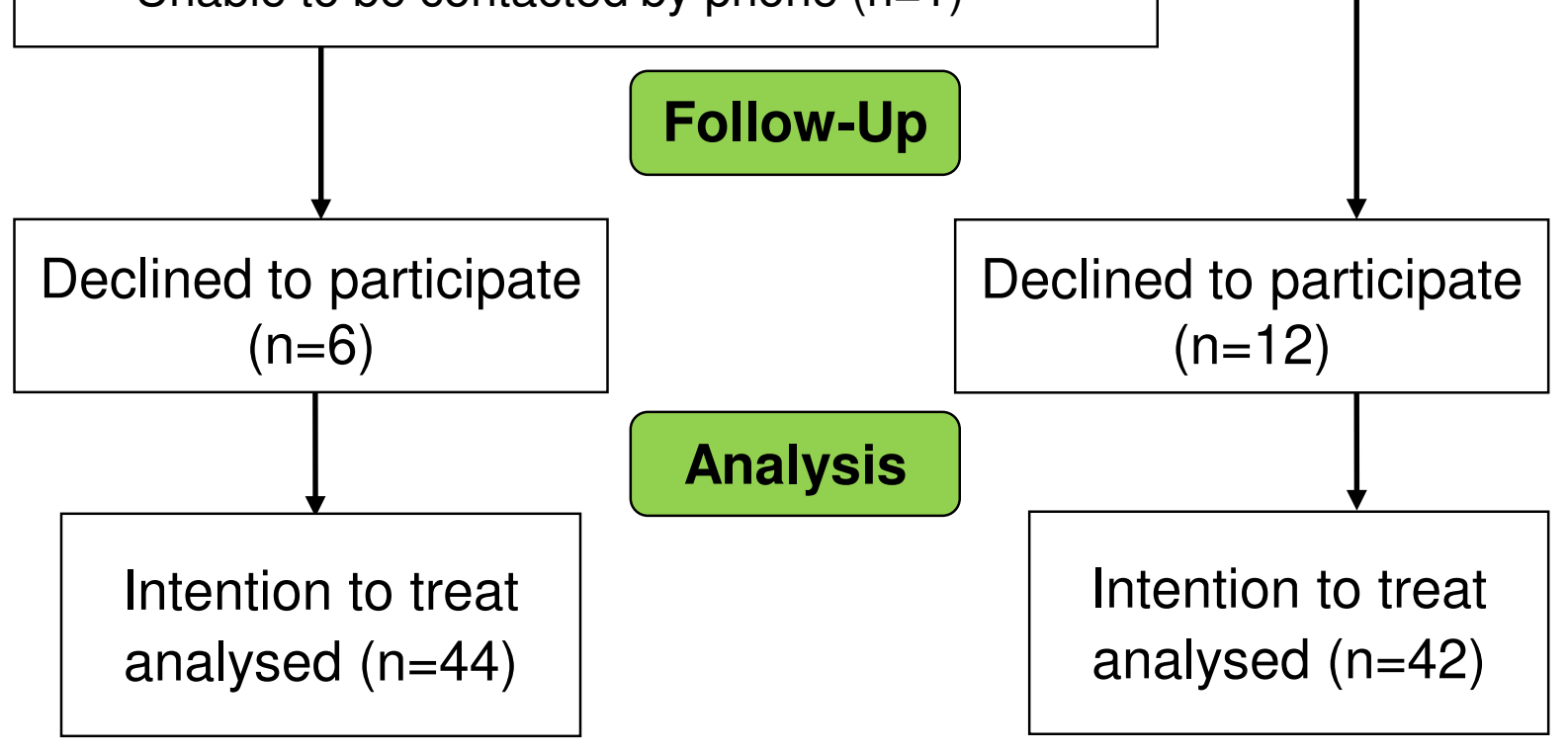

Results:

Wait-list control

$(\mathrm{n}=42)$

L

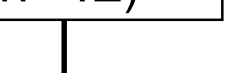

The average age of the CTI group was $66.6 \pm 5.5$ years old while that of the wait-list group was $67.5 \pm 6.4$ years old. At the baseline (T0), the TMSE scores of CTI and wait-list groups were similar (28.8 \pm 1.3 and $28.8 \pm 1.1$, respectively). All participants possessed a good quality of life with no depressive disorder. For the CTI group, the average number of cognitive training visits was 14.8 \pm 7.6 . At the primary endpoint, the statistical mean difference of TMSE was identified between CTI and wait-list groups by using intentionto-treat analysis ( $\triangle \mathrm{TMSE}$ between group at T1-T0 $=0.57 ; 95 \% \mathrm{Cl}=0.07$ to 1.08$)$. At the secondary endpoint, no significant difference of TMSE was found between the two groups ( $\triangle \mathrm{TMSE}$ between group at T2-T0 $=0.33 ; 95 \% \mathrm{Cl}=-0.23$ to 0.88 ). Additionally, at both endpoints, the two groups presented no notable discrepancy in six cognitive function subdomains, depression, and quality if life.

\section{Conclusion:}

CTI posed notable treatment effects (effect size; $d=0.56$ ) on the improvement of global cognitive function in healthy elderly Thais but did not demonstrate carryover effects. 